\title{
Size Dependent Acoustical Properties of bcc Metal
}

\author{
R.R. YADAV* AND D.K. PANDEY \\ Physics Department, Allahabad University, Allahabad-211002, India
}

(Received December 13, 2004; revised version April 6, 2005)

The ultrasonic attenuation is evaluated at $300 \mathrm{~K}$ along [100], [110], and [111] directions for the characterization of bcc metal Ta. The size of the metal is considered in nanorange. Ultrasonic velocity, Grüneisen parameter and acoustic coupling constant that depend on second and third order elastic constants are calculated for determination of ultrasonic attenuation coefficient. Second and third order elastic constants of the bcc metal Ta at nanoscale at $300 \mathrm{~K}$ are also calculated starting with only two basic parameters. For the information about defects at nanoscale, the dislocation drag coefficients are calculated for the metal at different size along [100] orientation. The ultrasonic attenuation increases with the size of material as the size variation of the thermal conductivity and the thermal relaxation time. There is significant increase in the attenuation up to $150 \mathrm{~nm}$.

PACS numbers: 62.20.Dc, 62.65.+k, 63.20.Kr

\section{Introduction}

Nanostructured materials have attracted much attention in the past decades due to their size dependent unique mechanical, physical, and chemical properties [1]. One of the principal differences between nanostructured materials and bulk ones is that more defects such as vacancies, voids, grain boundaries, and dislocations exist in nanostructured materials. The materials microstructure and associated physical properties are well related to the ultrasonic attenuation, velocity, and their related parameters $[2-5]$ that can be used to give insight into material.

*corresponding author; e-mail: rryadav1@rediffmail.com 
The bcc structured metal Ta has many applications such as that it can be used as replacement for platinum for laboratory apparatus, which has to give good corrosion resistance. The fluids in the human body do not react with the metal and hence it is used for surgical implants without rejection. Other applications include the use of tantalum carbide in cemented carbides that are used as cutting tools. The pure metal is used in the electronic industry in the manufacture of various types of electronic equipment as rectifiers, capacitors, lamp filaments etc. It is also used in vacuum systems as it has high absorption rate for residual gases and it forms alloys with nickel, molybdenum, which have good corrosion resistance, strength, and ductility.

Many of the thermophysical, microstructural properties of bcc metal Ta were studied. But so far ultrasonic properties at nanoscale are not reported in the literature. Therefore in the present investigation ultrasonic properties of this metal are determined at the nanoscale. We have calculated the second and third order elastic constants, non-linearity parameters and thermal relaxation time at nanoscale and at room temperature for the determination of ultrasonic attenuation and velocity. Dislocation parameters are calculated using their relation to different physical properties for the study of imperfection.

\section{Theory}

\subsection{Theory of elastic constants}

The elastic constant of the $n$-th order is defined as [6]:

$C_{i j k l m n}=\left(\partial^{n} F / \partial \eta_{i j} \partial \eta_{k l} \partial \eta_{m n}\right)$.

Here $F$ is the free energy density of undeformed material and $\eta_{i j}$ is Lagrangian strain components tensor. The Voigt notation (which has been adopted in the present investigation) $C_{I J K \ldots}$ has been used instead of tensor notation $C_{i j k l m n \ldots}$ for notation of elastic constants, for using $(i j) \rightarrow I$, according to the scheme $(11) \rightarrow 1$ etc. and $(23) \rightarrow 4$ etc. for cubic crystals; due to symmetry only three independent second-order elastic constants (SOEC) and six third-order elastic constants (TOEC) exist. The total free energy density $F$ is expanded in terms of strain $\eta$ and can be written (using Taylor's series expansion) as

$$
F=\sum_{n=0}^{\infty} F_{n}=\sum_{n=0}^{\infty} \frac{1}{n !}\left(\partial^{n} F / \partial \eta_{i j} \partial \eta_{k l} \partial \eta_{m n}\right) \eta_{i j} \eta_{k l} \eta_{m n}
$$

Hence free energy density in square and cubic terms of strain can be written $[6,7]$ as

$$
\begin{aligned}
& F_{2}+F_{3}=\frac{1}{2 !} C_{i j k l} \eta_{i j} \eta_{k l}+\frac{1}{3 !} C_{i j k l m n} \eta_{i j} \eta_{k l} \eta_{m n} \\
& F_{2}+F_{3}=(1 / 2) C_{11}\left(\eta_{11}^{2}+\eta_{22}^{2}+\eta_{33}^{2}\right)+C_{12}\left(\eta_{11} \eta_{22}+\eta_{11} \eta_{33}+\eta_{22} \eta_{33}\right)
\end{aligned}
$$




$$
\begin{aligned}
& +2 C_{44}\left(\eta_{12}^{2}+\eta_{23}^{2}+\eta_{31}^{2}\right)+(1 / 6) C_{111}\left(\eta_{11}^{3}+\eta_{22}^{3}+\eta_{33}^{3}\right) \\
& +(1 / 2) C_{112}\left[\eta_{11}^{2}\left(\eta_{22}+\eta_{33}\right)+\eta_{22}^{2}\left(\eta_{33}+\eta_{11}\right)+\eta_{33}^{2}\left(\eta_{11}+\eta_{22}\right)\right] \\
& +C_{123} \eta_{11} \eta_{22} \eta_{33}+2 C_{144}\left(\eta_{11} \eta_{23}^{2}+\eta_{22} \eta_{31}^{2}+\eta_{33} \eta_{12}^{2}\right) \\
& +2 C_{166}\left[\eta_{12}^{2}\left(\eta_{11}+\eta_{22}\right)+\eta_{23}^{2}\left(\eta_{22}+\eta_{33}\right)+\eta_{31}^{2}\left(\eta_{33}+\eta_{11}\right)\right] \\
& +8 C_{456} \eta_{12} \eta_{23} \eta_{31}
\end{aligned}
$$

where $C_{I J}$ are SOEC and $C_{I J K}$ are TOEC.

The free energy of a crystal containing $N$ cells at a finite temperature $T$ is given as [7]:

$$
F=V_{\mathrm{C}} N U+F^{\mathrm{Vib}},
$$

where $V_{\mathrm{C}}$ is the volume of the elementary cell, $U$ is the internal energy density of the crystal when all ions are in rest on their lattice point and $F^{\mathrm{Vib}}$ is the vibration free energy. Thus an elastic constant can be separated into two pairs

$$
C_{I J K \ldots}=C_{I J K \ldots}^{0}+C_{I J K \ldots}^{\mathrm{Vib}}
$$

The first and second ones are strain derivative of $U$ and $F^{\mathrm{Vib}}$ and they represent the static and vibrational elastic constants.

The expression of $U$ is expressed as

$$
U=\left(2 V_{\mathrm{C}}\right)^{-1} \sum_{\nu=1}^{2} \sum_{\left(\begin{array}{c}
m \\
\mu
\end{array}\right) \neq\left(\begin{array}{c}
0 \\
\nu
\end{array}\right)} \phi_{\mu \nu}\left(R_{\mu \nu}^{m 0}\right) \cong\left(2 V_{\mathrm{C}}\right)^{-1} \sum \phi_{\mu \nu}(R) .
$$

Here $R_{\mu \nu}^{m 0}$ is the distance between $\nu$-th ion in 0 -th cell and $\mu$-th ion in $m$-th cell and $\phi_{\mu \nu}(R)$ is the interaction potential formed by Coulombic and non-Coulombic parts

$$
\phi_{\mu \nu}(R)=\phi_{\mu \nu}^{\mathrm{C}}(R)+\phi_{\mu \nu}^{\mathrm{NC}}(R),
$$

where $\phi_{\mu \nu}^{\mathrm{C}}(R)= \pm e^{2} / R$ and $\phi_{\mu \nu}^{\mathrm{NC}}(R)=A \exp (-R / b)$. Here $e$ is electronic charge, \pm sign indicates for like and unlike charges, $A$ is constant and $b$ is hardness parameter.

When the crystal is deformed homogeneously the distances between the $(\nu, 0)$ ion and the $(\mu, m)$ ion in the deformed $\left(R_{\mu \nu}^{m 0}\right)$ and the undeformed states $\left(r_{\mu \nu}^{m 0}\right)$ are related to the Lagrangian strain component as

$$
\left(R_{\mu \nu}^{m 0}\right)^{2}-\left(r_{\mu \nu}^{m 0}\right)^{2}=2 \xi_{\mu \nu i}^{m 0} \xi_{\mu \nu j}^{m 0} \eta_{i j}=2 \rho_{\mu \nu}^{m 0},
$$

where $\xi_{\mu \nu i}^{m 0}$ is the $i$-th Cartesian component of the wector $r_{\mu \nu}^{m 0}$. This equation also defines the quantity $\rho_{\mu \nu}^{m 0}$. The energy $U$ given by Eq. (4) can be expanded in terms of $\rho$ up to cubic terms as 


$$
\begin{aligned}
U_{2}+ & U_{3}=\left(2 V_{\mathrm{C}}\right)^{-1} \sum\left[\left(\frac{\rho^{2}}{2 !}\right) \mathrm{D}^{2} \phi(R)\right]_{R=r}+\left(2 V_{\mathrm{C}}\right)^{-1} \sum\left[\left(\frac{\rho^{3}}{3 !}\right) \mathrm{D}^{3} \phi(R)\right]_{R=r} \\
& =\left(4 V_{\mathrm{C}}\right)^{-1}\left[\eta_{i j} \eta_{k l} \sum \xi_{i} \xi_{j} \xi_{k} \xi_{l} \mathrm{D}^{2} \phi(R)\right]_{R=r} \\
& +\left(12 V_{\mathrm{C}}\right)^{-1}\left[\eta_{i j} \eta_{k l} \eta_{m n} \sum \xi_{i} \xi_{j} \xi_{k} \xi_{l} \xi_{m} \xi_{n} \mathrm{D}^{3} \phi(R)\right]_{R=r}
\end{aligned}
$$

with abbreviation $\mathrm{D}=\frac{1}{R} \frac{\mathrm{d}}{\mathrm{d} R}$. Comparing Eq. (5) with Eq. (2) in reference to Eq. (3), the static component of elastic constants can be written as

$$
\begin{aligned}
& C_{11}^{0}=\left(2 V_{\mathrm{C}}\right)^{-1}\left[\sum \xi_{1}^{4} \mathrm{D}^{2} \phi(R)\right]_{R=r}, \\
& C_{12}^{0}=C_{44}^{0}=\left(2 V_{\mathrm{C}}\right)^{-1}\left[\sum \xi_{1}^{2} \xi_{2}^{2} \mathrm{D}^{2} \phi(R)\right]_{R=r}, \\
& C_{111}^{0}=\left(2 V_{\mathrm{C}}\right)^{-1}\left[\sum \xi_{1}^{6} \mathrm{D}^{3} \phi(R)\right]_{R=r}, \\
& C_{112}^{0}=C_{166}^{0}=\left(2 V_{\mathrm{C}}\right)^{-1}\left[\sum \xi_{1}^{4} \xi_{2}^{2} \mathrm{D}^{3} \phi(R)\right]_{R=r}, \\
& C_{123}^{0}=C_{144}^{0}=C_{456}^{0}=\left(2 V_{\mathrm{C}}\right)^{-1}\left[\sum \xi_{1}^{2} \xi_{2}^{2} \xi_{3}^{2} \mathrm{D}^{3} \phi(R)\right]_{R=r} .
\end{aligned}
$$

The elementary cell is supposed to have a cube edge of $2 r_{0}$, hence the volume becomes as $4\left(r_{0}\right)^{3}$. The nearest neighbour distance is $r_{1}=\sqrt{3} r_{0}$. The summation is done up to second neighbourhood whose coordinates are $( \pm 1, \pm 1, \pm 1) r_{0}, \quad( \pm 2,0,0) r_{0}, \quad(0, \pm 2,0) r_{0}$ and $(0,0, \pm 2) r_{0}$. The obtained expression for second and third order elastic constants at $0 \mathrm{~K}$ using above equations are as

$$
\begin{aligned}
& C_{11}^{0}=\frac{3}{8} \frac{e^{2}}{r_{0}^{4}} S_{5}^{(2)}+\frac{3 \phi\left(r_{1}\right)}{b r_{0}}\left(\frac{\sqrt{3}}{3 r_{0}}+\frac{1}{b}\right)+\frac{2 \phi\left(r_{2}\right)}{b r_{0}}\left(\frac{1}{2 r_{0}}+\frac{1}{b}\right), \\
& C_{12}^{0}=C_{44}^{0}=\frac{3}{8} \frac{e^{2}}{r_{0}^{4}} S_{5}^{1,1}+\frac{\phi\left(r_{2}\right)}{b r_{0}}\left(\frac{1}{2 r_{0}}+\frac{1}{b}\right), \\
& C_{111}^{0}=-\frac{15}{8} \frac{e^{2}}{r_{0}^{4}} S_{7}^{(3)}-\frac{\phi\left(r_{1}\right)}{9 b}\left(\frac{\sqrt{3}}{r_{0}^{2}}+\frac{3}{b r_{0}}+\frac{\sqrt{3}}{b^{2}}\right)-\frac{\phi\left(r_{2}\right)}{2 b}\left(\frac{3}{r_{0}^{2}}+\frac{6}{b r_{0}}+\frac{4}{b^{2}}\right), \\
& C_{112}^{0}=C_{166}^{0}=-\frac{15}{8} \frac{e^{2}}{r_{0}^{4}} S_{7}^{(2,1)}-\frac{\phi\left(r_{1}\right)}{9 b}\left(\frac{\sqrt{3}}{r_{0}^{2}}+\frac{3}{b r_{0}}+\frac{\sqrt{3}}{b^{2}}\right), \\
& C_{123}^{0}=C_{456}^{0}=C_{144}^{0}=-\frac{15}{8} \frac{e^{2}}{r_{0}^{4}} S_{7}^{(1,1,1)}-\frac{\phi\left(r_{1}\right)}{9 b}\left(\frac{\sqrt{3}}{r_{0}^{2}}+\frac{3}{b r_{0}}+\frac{\sqrt{3}}{b^{2}}\right),
\end{aligned}
$$

where lattice sums are 


$$
\begin{aligned}
& S_{1}^{0}=-Z_{0}=-1.017678, \quad S_{5}^{(2)}=0.354190, \quad S_{5}^{(1,1)}=0.346708 \\
& S_{7}^{(3)}=0.540901, \quad S_{7}^{(2,1)}=-0.093356, \quad S_{7}^{(1,1,1)}=-0.159996
\end{aligned}
$$

and $\phi\left(r_{1}\right)=A \exp \left(-r_{1} / b\right), \phi\left(r_{2}\right)=A \exp \left(-r_{2} / b\right), r_{1}=\sqrt{3} r_{0}, r_{2}=2 r_{0}$. The value of $A$ is obtained from the equilibrium condition, given as

$$
A=\left(b Z_{0} e^{2} / r_{0}^{2}\right)\left[8 \sqrt{3} \exp \left(-r_{1} / b\right)+12 \exp \left(-r_{2} / b\right)\right] .
$$

The elastic constants due to vibrational free energy component is given by [8]:

$$
C_{I J K \ldots}^{\mathrm{Vib}}=a_{I J K \ldots T}
$$

Here

$$
\begin{aligned}
a_{I J K \ldots}=l_{1} k_{\mathrm{B}}\left|\frac{\partial C_{I J K \ldots}^{0}}{\partial r}\right|_{r=r_{0}}+\frac{f_{I J K \ldots}^{\mathrm{Vib}}}{T V_{\mathrm{C}}} \\
l_{1}=-r_{0}\left[\frac{8}{3}\left(2 \rho_{1}+2 \rho_{1}^{2}-\rho_{1}^{3}\right) \phi\left(r_{1}\right)+\frac{3}{2}\left(2 \rho_{2}+2 \rho_{2}^{2}-\rho_{2}^{3}\right) \phi\left(r_{2}\right)\right] Y^{-1}, \\
Y=\left[\frac{8}{3}\left(\rho_{1}^{2}-2 \rho_{1}\right) \phi\left(r_{1}\right)+\frac{3}{2}\left(\rho_{2}^{2}-2 \rho_{2}\right) \phi\left(r_{2}\right)\right] \\
\times\left[\frac{8}{3}\left(\rho_{1}^{2}-2 \rho_{1}\right) \phi\left(r_{1}\right)+2\left(\rho_{2}^{2}-2 \rho_{2}\right) \phi\left(r_{2}\right)\right]
\end{aligned}
$$

where $\rho_{1}=r_{1} / b, \rho_{2}=r_{2} / b, k_{\mathrm{B}}$ is the Boltzmann constant and $f_{I J K \ldots}^{\mathrm{Vib}}$ is vibrational free energy per unit cell that is given by the following expressions:

$$
\begin{aligned}
f_{11}^{\mathrm{Vib}} & =\frac{k_{\mathrm{B}} T}{4}\left(G_{2}-\frac{G_{1}^{2}}{6}\right), \quad f_{12}^{\mathrm{Vib}}=\frac{k_{\mathrm{B}} T}{4}\left(G_{1,1}-\frac{G_{1}^{2}}{6}\right), \\
f_{44}^{\mathrm{Vib}} & =\frac{k_{\mathrm{B}} T}{4} G_{1,1}, \quad f_{111}^{\mathrm{Vib}}=\frac{k_{\mathrm{B}} T}{4}\left(G_{3}-\frac{1}{2} G_{1} G_{2}+\frac{G_{1}^{3}}{18}\right), \\
f_{112}^{\mathrm{Vib}} & =\frac{k_{\mathrm{B}} T}{4}\left(G_{2,1}-\frac{1}{3} G_{1,1} G_{1}-\frac{1}{6} G_{2} G_{1}+\frac{G_{1}^{3}}{18}\right), \\
f_{123}^{\mathrm{Vib}} & =\frac{k_{\mathrm{B}} T}{4}\left(G_{1,1,1}-\frac{1}{2} G_{1,1} G_{1}+\frac{G_{1}^{3}}{18}\right), \quad f_{144}^{\mathrm{Vib}}=\frac{k_{\mathrm{B}} T}{4}\left(G_{1,1,1}-\frac{1}{2} G_{1,1} G_{1}\right), \\
f_{166}^{\mathrm{Vib}} & =\frac{k_{\mathrm{B}} T}{4}\left(G_{1,1,1}-\frac{1}{2} G_{1,1} G_{1}\right), \quad f_{456}^{\mathrm{Vib}}=\frac{k_{\mathrm{B}} T}{4} G_{1,1,1}, \\
G_{1} & =\left[\frac{8}{9} \phi\left(r_{1}\right)\left(2 \rho_{1}+2 \rho_{1}^{2}-\rho_{1}^{3}\right)+\frac{1}{2} \phi\left(r_{2}\right)\left(2 \rho_{2}+2 \rho_{2}^{2}-\rho_{2}^{3}\right)\right] Z, \\
G_{2} & =\left[\frac{8}{27} \phi\left(r_{1}\right)\left(-6 \rho_{1}-6 \rho_{1}^{2}-\rho_{1}^{3}+\rho_{1}^{4}\right)+\frac{1}{2} \phi\left(r_{2}\right)\left(-6 \rho_{2}-6 \rho_{2}^{2}-\rho_{2}^{3}+\rho_{3}^{4}\right)\right] Z,
\end{aligned}
$$




$$
\begin{aligned}
G_{1,1}= & {\left[\frac{8}{27} \phi\left(r_{1}\right)\left(-6 \rho_{1}-6 \rho_{1}^{2}-\rho_{1}^{3}+\rho_{1}^{4}\right)\right] Z, } \\
G_{3}= & {\left[\frac{8}{81} \phi\left(r_{1}\right)\left(30 \rho_{1}+30 \rho_{1}^{2}+9 \rho_{1}^{3}-\rho_{1}^{4}-\rho_{1}^{5}\right)\right.} \\
& \left.+\frac{1}{2} \phi\left(r_{2}\right)\left(30 \rho_{2}+30 \rho_{2}^{2}+9 \rho_{2}^{3}-\rho_{2}^{4}-\rho_{2}^{5}\right)\right] Z, \\
G_{2,1}= & G_{1,1,1}=\left[\frac{8}{81} \phi\left(r_{1}\right)\left(30 \rho_{1}+30 \rho_{1}^{2}+9 \rho_{1}^{3}-\rho_{1}^{4}-\rho_{1}^{5}\right)\right] Z, \\
Z= & {\left[\frac{4}{9} \phi\left(r_{1}\right)\left(\rho_{1}^{2}-2 \rho_{1}\right)+\frac{1}{4} \phi\left(r_{2}\right)\left(\rho_{2}^{2}-\rho_{2}\right)\right] }
\end{aligned}
$$

Using above expressions, the vibrational part of elastic components have been calculated by Eq. (6), whose addition to static part of elastic constants gives the elastic constants at a particular temperature. All the calculations are done with the help of programming in $\mathrm{C}^{++}$.

\subsection{Theory of attenuation}

For the evaluation of ultrasonic attenuation coefficient, we have used the phonon-phonon interaction mechanism given by Mason and Bateman [9, 10]. It is more genuine theory for studying the anharmonicity of crystals as it directly involves elastic constants through non-linearity parameters $D$ in the evaluation of ultrasonic attenuation coefficient $\alpha$. The ultrasonic attenuation over frequency square $\left(\alpha / f^{2}\right)_{\text {Akh }}$ (Akhieser type loss) due to phonon-phonon interaction mechanism at $\omega \tau \ll 1[11]$ is expressed as

$$
\left(\alpha / f^{2}\right)_{\mathrm{Akh}}=\frac{E_{0}(D / 3) 4 \pi^{2} \tau}{2 d V^{3}},
$$

where

$$
D=9\left\langle\left(\gamma_{i}^{j}\right)^{2}\right\rangle-\frac{3\left\langle\gamma_{i}^{j}\right\rangle^{2} C_{V} T}{E_{0}} .
$$

Here $D$ and $V$ is the non-linearity parameter and velocity of ultrasonic wave for longitudinal and shear wave. $E_{0}$ and $C_{V}$ are thermal energy density and the specific heat per unit volume, respectively. The $\left\langle\gamma_{i}^{j}\right\rangle$ is the average Grüneisen number, $j$ is the direction of propagation and $i$ is the mode of propagation. $\left\langle\gamma_{i}^{j}\right\rangle$ is related to SOEC/TOEC [9]. $d$ is density of material and $f$ is frequency of the wave. The thermal relaxation time $(\tau)$, which is equal to thermal relaxation time for shear wave $\left(\tau_{\mathrm{S}}\right)$ and is half of thermal relaxation time for longitudinal wave $\left(\tau_{\mathrm{L}}\right)$ is twice that of shear wave and is written as 


$$
\tau=\tau_{\mathrm{S}}=\frac{1}{2} \tau_{\mathrm{L}}=\frac{3 K}{C_{V}(\bar{V})^{2}}
$$

where $K$ is thermal conductivity and $\bar{V}$ is the Debye average velocity of ultrasonic wave as

$$
\begin{aligned}
& \frac{3}{(\bar{V})^{3}}=\frac{1}{V_{\mathrm{L}}^{3}}+\frac{2}{V_{\mathrm{S}}^{3}}, \\
& V_{\mathrm{L}}=\sqrt{\frac{C_{11}}{d}} \quad \text { and } \quad V_{\mathrm{S}}=\sqrt{\frac{C_{44}}{d}}
\end{aligned}
$$

where $V_{\mathrm{L}}$ and $V_{\mathrm{S}}$ are the velocity of longitudinal and shear wave, respectively.

The thermal conductivity $(K)$ depends on the size of material [12]:

$$
\frac{1}{K}=\frac{2}{n k_{\mathrm{B}} u}\left(\frac{1}{l_{\infty}}+\frac{4}{L}\right)
$$

Here $n$ - the number density of the atoms, $k_{\mathrm{B}}$ - the Boltzmann constant, $u$ - the group velocity of acoustic band, $l_{\infty}$ - the phonon mean free path in an infinite system and $L$ is the system size. The number density $n$ in an fcc system is $4 / d_{0}^{3}$ and in the bcc system $2 / d_{0}^{3}$, where $d_{0}$ is the lattice constant.

The propagation of longitudinal ultrasonic wave creates compression and rarefaction throughout the lattice. The rarefied regions are colder than that of compressed regions. Thus there is flow of heat between these two regions. Hence, thermoelastic loss $\left(\alpha / f^{2}\right)$ th occurs and is obtained by expression [11]:

$$
\left(\alpha / f^{2}\right)_{\mathrm{th}}=\frac{4 \pi^{2}\left\langle\gamma_{i}^{j}\right\rangle^{2} K T}{2 d V_{\mathrm{L}}^{5}} .
$$

\subsection{Theory of imperfection}

Dislocation drag is a parameter for which the phonon-phonon interaction can produce appreciable effect on the motion of linear imperfections in a lattice through the phenomenon of drag. The thermal losses due to such a motion can be computed by multiplying the following drag coefficients by the square of the dislocation velocity:

$$
\begin{aligned}
& B_{\text {screw }}=0.071 \varepsilon, \\
& B_{\text {edge }}=\frac{0.053 \varepsilon}{1-\sigma^{2}}+\frac{0.0079}{1-\sigma^{2}}\left(\frac{\mu}{B}\right) \chi,
\end{aligned}
$$

where

$$
\begin{aligned}
& \chi=\varepsilon_{\mathrm{L}}-\frac{4}{3} \varepsilon_{\mathrm{S}}, \quad \varepsilon_{\mathrm{L}}=\frac{E_{0} D_{\mathrm{L}} \tau_{\mathrm{L}}}{3}, \quad \varepsilon_{\mathrm{S}}=\frac{E_{0} D_{\mathrm{S}} \tau_{\mathrm{S}}}{3}, \quad B=\frac{1}{3}\left(C_{11}+2 C_{12}\right), \\
& \mu=\frac{1}{3}\left(C_{11}-C_{12}+C_{44}\right) \quad \text { and } \quad \sigma=\frac{C_{12}}{C_{11}+C_{12}} .
\end{aligned}
$$


$\mu, \varepsilon, \sigma, B$, and $\chi$ are the shear modulus, phonon viscosity, Poisson's ratio, bulk modulus, and compressional viscosity, respectively. $C_{11}, C_{12}$ and $C_{44}$ are the SOEC for cubic metals.

\section{Results}

3.1. Second and third order elastic constants at $300 \mathrm{~K}$

Calculated values of SOEC and TOEC for Ta metal at $300 \mathrm{~K}$ and at nanoscale are presented in Table I.

TABLE I

SOEC and TOEC $\left(10^{11} \mathrm{~N} / \mathrm{m}^{2}\right)$ of metal Ta at $300 \mathrm{~K}$ and at nanoscale.

\begin{tabular}{c|c|c|c|c|c|c|c|c|c}
\hline \hline Metal & $C_{11}$ & $C_{12}$ & $C_{44}$ & $C_{111}$ & $C_{112}$ & $C_{123}$ & $C_{144}$ & $C_{166}$ & $C_{456}$ \\
\hline Ta & 2.711 & 0.318 & 0.319 & -20.301 & -10.727 & -8.898 & -6.469 & -6.988 & -8.676
\end{tabular}

Ultrasonic longitudinal and shear wave velocities are calculated using the values of second order elastic constants and the Debye average velocity $(\bar{V}))$ is calculated using Eq. (10). The thermal energy density $\left(E_{0}\right)$, density $(\rho)$, and specific heat per unit volume $\left(C_{V}\right)$ are calculated using physical constant table and the Debye temperature. The value of $d, \bar{V}, E_{0}$, and $C_{V}$ are presented in Table II.

TABLE II

Density $(d)$, specific heat per unit volume $\left(C_{V}\right)$, energy density $\left(E_{0}\right)$, Debye average velocity $(\bar{V})$ of Ta metal at $300 \mathrm{~K}$.

\begin{tabular}{c|c|c|c|c}
\hline \hline Metal & $d\left[10^{3} \mathrm{~kg} / \mathrm{m}^{3}\right]$ & $C_{V}\left[10^{6} \mathrm{~J} /\left(\mathrm{m}^{3} \mathrm{~K}\right)\right]$ & $E_{0}\left[10^{8} \mathrm{~J} / \mathrm{m}^{3}\right]$ & $\bar{V}\left[10^{3} \mathrm{~m} / \mathrm{s}\right]$ \\
\hline Ta & 16.66 & 2.24 & 5.118 & 1.555
\end{tabular}

\subsection{Thermal relaxation time, Grüneisen numbers and non-linearity parameters}

The thermal conductivity data $(K)$ in size range $10-300 \mathrm{~nm}$ are taken from the literature [12]. The thermal relaxation time are determined using thermal conductivity with Eq. (9). The size dependent value of $K$ and $\tau$ are presented in Table III. The size variation of $\tau$ with fitted curve is shown in Fig. 1.

The Grüneisen parameters are calculated using SOEC and TOEC and acoustic coupling constants $\left(D_{\mathrm{L}}\right.$ and $D_{\mathrm{S}}$ ) for longitudinal and shear wave are estimated along different orientations [100], [110] and [111] at $300 \mathrm{~K}$ for Ta metal at the nanoscale using Eq. (8). The calculated values of the Grüneisen parameters and acoustic coupling constants for Ta at $300 \mathrm{~K}$ along different directions are presented in Table IV. 
TABLE III

Thermal conductivity $(K)$, thermal relaxation time $(\tau)$ of Ta metal at $300 \mathrm{~K}$ in size range $10-300 \mathrm{~nm}$.

\begin{tabular}{c|c|c|c|c|c|c}
\hline \hline Size $[\mathrm{nm}]$ & 10 & 20 & 40 & 80 & 160 & 300 \\
\hline$K[\mathrm{~W} /(\mathrm{m} \mathrm{K})]$ & 2.43 & 4.17 & 5.88 & 9.89 & 14.29 & 16.67 \\
$\tau\left[10^{-12} \mathrm{~s}\right]$ & 1.345 & 2.308 & 3.255 & 5.475 & 7.910 & 9.228
\end{tabular}

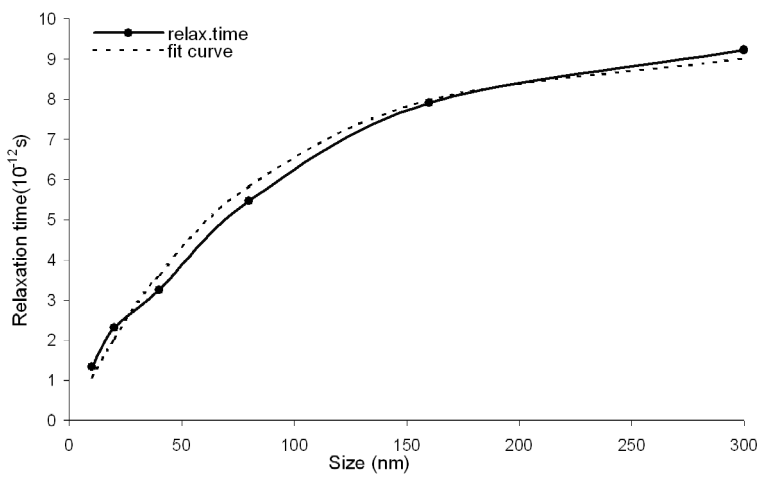

Fig. 1. Relaxation time vs. size of metal Ta.

TABLE IV

Average Grüneisen number $\left\langle\gamma_{i}^{j}\right\rangle_{\mathrm{L}}$ for longitudinal wave, average square Grüneisen number $\left\langle\left(\gamma_{i}^{j}\right)^{2}\right\rangle_{\mathrm{L}}$ and $\left\langle\left(\gamma_{i}^{j}\right)^{2}\right\rangle_{\mathrm{S}}$ for longitudinal and shear wave, non-linearity parameters $D_{\mathrm{L}}$, $D_{\mathrm{S}}$ for longitudinal and shear wave of metal Ta at $300 \mathrm{~K}$.

\begin{tabular}{l|c|c|c|c|c}
\hline \hline Direction & $\left\langle\gamma_{i}^{j}\right\rangle_{\mathrm{L}}$ & $\left\langle\left(\gamma_{i}^{j}\right)^{2}\right\rangle_{\mathrm{L}}$ & $\left\langle\left(\gamma_{i}^{j}\right)^{2}\right\rangle_{\mathrm{S}}$ & $D_{\mathrm{L}}$ & $D_{\mathrm{S}}$ \\
\hline$[100]$ & 1.936 & 20.396 & 11.637 & 168.799 & 104.737 \\
{$[110]^{*}$} & -2.416 & 22.930 & 4.180 & 183.377 & 37.622 \\
{$[110]^{* *}$} & -2.416 & 22.930 & 0.907 & 183.377 & 8.161 \\
{$[111]^{* * *}$} & -1.523 & 7.276 & 3.513 & 146.344 & 31.618 \\
\hline
\end{tabular}

* polarized along [001] direction,

** polarized along $[1 \overline{1} 0]$ direction,

*** polarized along $[\overline{1} 10]$ direction.

\subsection{Ultrasonic attenuation coefficient}

Ultrasonic attenuation coefficient over frequency square $\left(\alpha / f^{2}\right)$ AkhLong for longitudinal wave, $\left(\alpha / f^{2}\right)_{\text {AkhShear }}$ for shear wave and thermoelastic loss $\left(\alpha / f^{2}\right)_{\text {th }}$ have been calculated using Eqs. (7) and (12) along different orientations for different nanosizes of metal Ta and are shown in Figs. 2-4. 


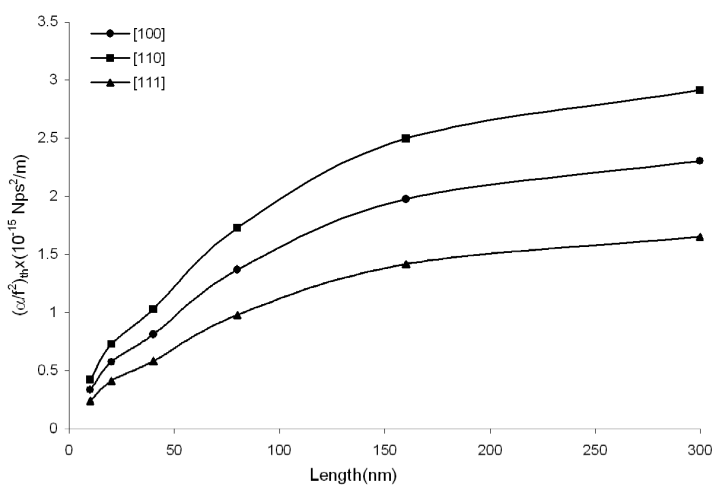

Fig. 2. $\left(\alpha / f^{2}\right)_{\text {th }}$ vs. size of metal Ta.

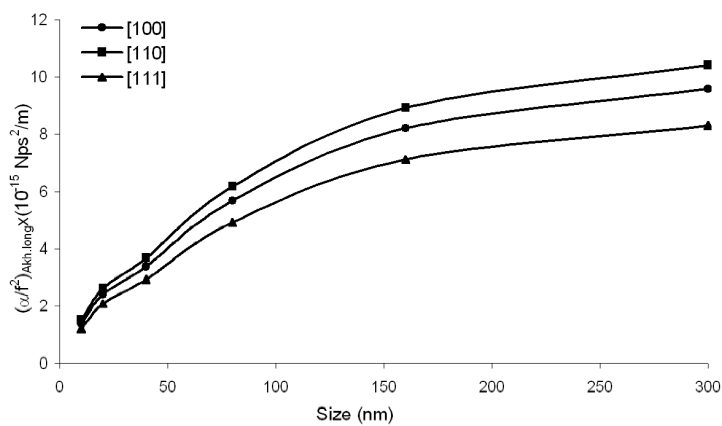

Fig. 3. $\left(\alpha / f^{2}\right)$ Akh.long vs. size of metal Ta.

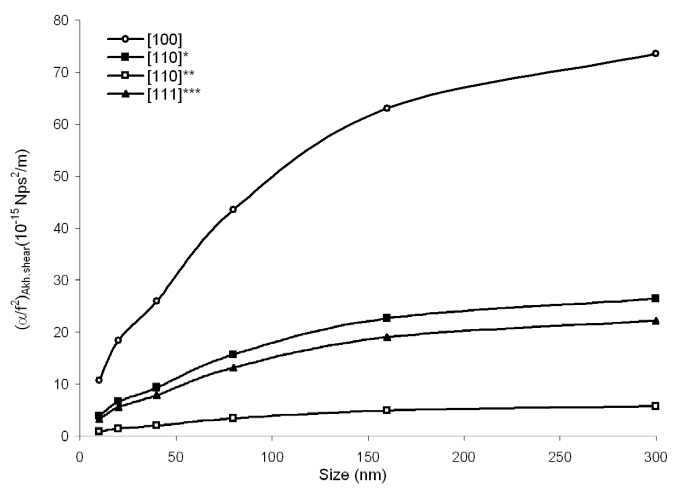

Fig. 4. $\left(\alpha / f^{2}\right)_{\text {Akh.shear }}$ vs. size of metal Ta. Designations of $*, * *, * * *$ as in Table IV. 


\subsection{Size dependence of dislocation drag coefficient}

Dislocation parameters $B_{\text {screw }}$ and $B_{\text {edge }}$ are calculated using Eqs. (13) and (14) for longitudinal and shear waves along [100] orientation for different nanosizes of Ta metal and are presented in Table $\mathrm{V}$.

TABLE V

$B_{\text {screw }}$ and $B_{\text {edge }}$ for longitudinal and shear wave along [100] orientation for the metal Ta from size 10-300 nm.

\begin{tabular}{c|c|c|c|c}
\hline \hline Size [nm] & $\begin{array}{c}\left(B_{\text {screw }}\right)_{\text {long }} \\
{[\mathrm{mP}]}\end{array}$ & $\begin{array}{c}\left(B_{\text {screw }}\right)_{\text {shear }} \\
{[\mathrm{mP}]}\end{array}$ & $\begin{array}{c}\left(B_{\text {edge }}\right)_{\text {long }} \\
{[\mathrm{mP}]}\end{array}$ & $\begin{array}{c}\left(B_{\text {edge }}\right)_{\text {shear }} \\
{[\mathrm{mP}]}\end{array}$ \\
\hline 10 & 55.00 & 17.06 & 54.89 & 19.54 \\
20 & 94.38 & 29.28 & 94.19 & 33.53 \\
40 & 133.10 & 41.29 & 132.84 & 47.28 \\
80 & 223.88 & 69.46 & 223.43 & 79.53 \\
160 & 323.46 & 100.35 & 322.80 & 114.90 \\
300 & 377.35 & 117.07 & 376.59 & 134.04
\end{tabular}

\section{Discussion}

We have made the calculations of SOEC and TOEC starting with only two basic parameters - lattice parameter and hardness parameter. Lattice parameter (nearest neighbour distance) decreases with size of the metal [13]. Lattice contraction leads to shift of X-ray diffraction peaks to higher diffraction angles. Therefore we have taken the smaller values of nearest neighbour distance for Ta metal at the nanoscale in comparison to normal crystalline size of the metal. The size variation of the lattice parameter is significant only from 10 to $30 \mathrm{~nm}$ [13]. There is only $0.02 \%$ change in the lattice parameter in the range. This small variation in the lattice parameter does not reflect any significant change in SOEC/TOEC and finally in the ultrasonic parameters of the metal at different nanosizes. Thus it is justified to suppose that the size variation of the lattice parameter is insignificant with respect to our calculations from 10 to $300 \mathrm{~nm}$ size of the metal.

The values of $C_{11}, C_{12}$, and $C_{44}$ at $5 \mathrm{~K}$ evaluated by present theory are $2.678 \times 10^{11} \mathrm{~N} / \mathrm{m}^{2}, 0.317 \times 10^{11} \mathrm{~N} / \mathrm{m}^{2}$, and $0.259 \times 10^{11} \mathrm{~N} / \mathrm{m}^{2}$, respectively. The values of $C_{11}, C_{12}$, and $C_{44}$ at $4.2 \mathrm{~K}$ in the work of Seraphim and Marcus [14] are $2.68 \times 10^{11} \mathrm{~N} / \mathrm{m}^{2}, 1.6 \times 10^{11} \mathrm{~N} / \mathrm{m}^{2}$ and $0.865 \times 10^{11} \mathrm{~N} / \mathrm{m}^{2}$, respectively. If we compare the value of $C_{11}$, then there is good agreement in present work and work of Seraphim and Marcus. However, there is small variation in magnitude of $C_{12}$ and $C_{44}$ values, while the order is the same. The present values of $C_{11}, C_{12}$, and $C_{44}$ at $300 \mathrm{~K}$ are $2.711 \times 10^{11} \mathrm{~N} / \mathrm{m}^{2}, 0.318 \times 10^{11} \mathrm{~N} / \mathrm{m}^{2}$, and $0.319 \times 10^{11} \mathrm{~N} / \mathrm{m}^{2}$. 
The values given by Sigalas and Papaconstantopoulos are $2.650 \times 10^{11} \mathrm{~N} / \mathrm{m}^{2}$, $1.590 \times 10^{11} \mathrm{~N} / \mathrm{m}^{2}$, and $0.82 \times 10^{11} \mathrm{~N} / \mathrm{m}^{2}$ [15]. The slight discrepancy in the values is obvious because our calculations are made at the nanoscale. On comparison of the methods, our present method of calculation is so simple. It involves only one basic parameter (lattice parameter) for bcc/fcc structure for the determination of SOEC and TOEC at different temperatures. On the other hand, the method by Sigalas and Papaconstantopoulos describes the determination of SOEC at room temperature and involves so many coefficients and parameters to be determined for the materials. Thus the present formulation for calculation of higher order elastic constants for bcc structured material is well justified.

A perusal of Figs. 2-4 shows that the ultrasonic attenuation normally increases with size of the metal. The variation of attenuation in $\mathrm{Ta}$ is significant from $10 \mathrm{~nm}$ to $150 \mathrm{~nm}$ and it is insignificant from $150 \mathrm{~nm}$ to $300 \mathrm{~nm}$. Now it is important to describe the behaviour of the physical properties, which are mainly contributing to the size dependence of the total ultrasonic attenuation.

As discussed, there is insignificant variation in lattice parameter and consequently in second and third order elastic constants from size 10-300 $\mathrm{nm}$ of the metal. Therefore, the ultrasonic attenuation is not much affected by the second and third order elastic constants of the metal at the nanoscale.

The Grüneisen parameters and acoustic coupling constants are the direct consequence of second and third order elastic constants, therefore these parameters also do not much affect the size dependence of ultrasonic attenuation in Ta at the nanoscale size. The values of these parameters are different for different propagating directions of the wave.

Another important property in the evaluation of ultrasonic attenuation is thermal relaxation time, which is computed with the help of size dependent thermal conductivity data. It is the time taken for the re-establishment of the thermal equilibrium after the interaction of thermal phonons and acoustical phonons. The variation of thermal relaxation time $(\tau)$ with the size of Ta metal is presented in Table III. A perusal of Fig. 1 shows that $\tau$ increases with size and its size dependence can be given by the relation $\tau=\tau_{0}\left(1-\mathrm{e}^{-x / \lambda}\right)$, where $x$ is the value of size of the metals and $\tau_{0}$ and $\lambda$ are some constants. In our evaluation, the thermoelastic loss over frequency square $\left(\alpha / f^{2}\right)_{\text {th }}$ is proportional to thermal conductivity as in Eq. (11) and the ultrasonic attenuation coefficient over frequency square $\left(\alpha / f^{2}\right)$ Akh for longitudinal and shear wave is proportional to thermal relaxation time as in Eq. (7). Thus the size variation of ultrasonic attenuation coefficient and thermoelastic loss follow the same nature as the size variation of thermal relaxation and thermal conductivity.

On quantitative comparison between the ultrasonic attenuation $\left(\alpha / f^{2}\right)$ at normal crystalline size [16] and the ultrasonic attenuation $\left(\alpha / f^{2}\right)$ in Ta at the nanoscale size, it is observed that the values of ultrasonic attenuation $\left(\alpha / f^{2}\right)$ in Ta at the nanoscale size are higher than the values at normal size, but the 
order of the values remains the same. The ratio of ultrasonic attenuation for shear to that for longitudinal wave are 4.3 (experimental) and 5.02 (theoretical) at normal size in the work of Levy et al. [17], while in our investigation this ratio at nanoscale is $\approx 7.68$. The comparison of the both investigations also implies the larger attenuation at nanoscale. Thus, in general some sort of imperfections are predicted on the basis of larger attenuation at nanoscale of the metal in comparison to the normal crystalline metal.

The dislocation drag coefficients $B_{\text {screw }}$ and $B_{\text {edge }}$ depend on phonon viscosity $(\varepsilon)$ as in Eq. (13) and (14) and phonon viscosity depends on relaxation time, which depends on size of the metal. Thus dislocation drag coefficients at different nanosizes of the metal are predominantly affected by thermal relaxation time. Therefore these coefficients increase with the size of metal. The values of these coefficients are higher than those at normal crystalline size. Thus more defects like dislocation movements may be predicted in the nanoscale size of the metal.

\section{Conclusions}

On the basis of above discussion, the present theory for the evaluation of second and third order elastic constants of bcc structured metal Ta is valid. Elastic constants (SOEC and TOEC) are not very important for size dependence of the ultrasonic attenuation in Ta at the nanoscale at $300 \mathrm{~K}$. The size dependence of ultrasonic attenuation coefficients and dislocation coefficient are predominantly affected by thermal relaxation time and thermal conductivity. The larger values of ultrasonic attenuation coefficients and dislocation coefficients at nanoscale reveal the fact that more imperfections exist at the nanosized material.

\section{Acknowledgments}

The work was financially supported by the Department of Science and Technology of Government of India (DST project no. SP/S2/M16/2001).

\section{References}

[1] Z. Bian, R.J. Wang, D.Q. Zhao, M.X. Pan, Z.X. Wang, W.H. Wang, Appl. Phys. Lett. 82, 2790 (2003).

[2] P.A. Elmore, M.A. Breazeale, Ultrasonics 41, 709 (2004).

[3] S. Mukherjee, C. Basu, U.S. Ghosh, J. Non-Cryst. Solids 144, 159 (1992).

[4] R.R. Yadav, D. Singh, Intermetallics 9, 189 (2001).

[5] S.K. Kor, R.R. Yadav, Kailash, J. Phys. Soc. Japan 55, 287 (1986).

[6] K. Brugger, Phys. Rev. A 133, 1611 (1964).

[7] S. Mori, Y. Hiki, J. Phys. Soc. Japan 45, 1449 (1978).

[8] P.B. Ghate, Phys. Rev. A 139, 1666 (1965). 
[9] W.P. Mason, Physical Acoustics, Vol. III B, Academic Press, New York 1965, p. 237.

[10] W.P. Mason, T.B. Bateman, J. Acoust. Soc. Am. 40, 852 (1966).

[11] R.R. Yadav, D. Singh, Acoust. Phys. 49, 595 (2003).

[12] P. Heino, E. Ristolainen, Microelectron. J. 34, 773 (2003).

[13] J. Woltersdorf, A.S. Nepuko, E. Pippel, Surf. Sci. 106, 64 (1981).

[14] D.P. Seraphim, P.M. Marcus, IBM J. 190, 94 (1962).

[15] M.M. Sigalas, D.A. Papaconstantopoulos, Phys. Rev. B 49, 1574 (1994).

[16] K. Shankar, Ph.D. thesis, University of Allahabad, Allahabad 1981, p. 114.

[17] M. Levy, R. Kagiwada, I. Rudnick, Phys. Rev. 132, 2039 (1963). 